\title{
GLI AMMINISTRATORI LOCALI \\ DI FORZA ITALIA
}

\author{
di Alessandro Tonarelli
}

\section{Introduzione}

A pochi mesi dal primo congresso nazionale di Forza Italia riteniamo utile esporre, sia pure in forma sintetica, $i$ risultati di una ricerca empirica sugli eletti di questo partito nei consigli comunali, provinciali e regionali ${ }^{1}$. La decisione di focalizzare l'analisi sui rappresentanti di Forza Italia nelle amministrazioni locali è suggerita da una serie di fattori, alcuni di rilievo sistemico e altri di portata più circoscritta. I primi inducono a collocare lo studio dei gruppi dirigenti locali di Forza Italia nel più ampio contesto della crisi e del cambiamento del sistema partitico italiano. A tale proposito, posto che fra gli indicatori più importanti di questo fenomeno troviamo il mutamento organiz-

1 Questo scritto si basa sui risultati di un sondaggio postale realizzato nel periodo aprile-settembre 1997. Il campione è composto da 167 consiglieri regionali, cioè tutti gli iscritti ai gruppi consiliari di FI nelle varie regioni d'Italia con l'eccezione della Valle d'Aosta e del Trentino-Alto Adige; 97 consiglieri provinciali (tutti i capigruppo consiliari delle amministrazioni ove era presente FI); 250 consiglieri comunali selezionati per campionamento casuale, tenendo ferma la stratificazione territoriale su tre macro-aree (Nord, Centro e Sud) fra tutti gli eletti di Forza Italia nei comuni italiani superiori ai 15.000 abitanti. Per ogni consigliere sono stati realizzati due recall postali a distanza di circa un mese l'uno dall'altro. Il totale dei questionari inviati è di 514 ; i rientri sono stati 160 per una redemption complessiva del $31,1 \%$. Tale cifra costituisce il totale dei casi analizzati nelle elaborazioni qui presentate ed è pari all' $8,8 \%$ dell'universo di riferimento costituito, come dichiarato da Forza Italia, da 1.815 unità fra consiglieri regionali, provinciali e comunali (centri al di sopra dei 15.000 abitanti). La ricerca è stata finanziata dal Consiglio Nazionale delle Ricerche - Comitato per le Scienze Giuridiche e Politiche nell'ambito del progetto «Partiti e sistemi di partito in Italia. Le trasformazioni organizzative» diretto da Leonardo Morlino. Al Cnr ed a tutti gli amici che a vario titolo hanno collaborato alla realizzazione della ricerca ed alla scrittura dell'articolo (Liborio Mattina, Leonardo Morlino, Caterina Paolucci, Luca Moreschini), va il sentito ringraziamento dell'autore. 
zativo ed il ricambio delle élites (Morlino 1996), uno studio del caso come quello condotto consente di misurare, con riferimento ad un importante attore politico, l'intensità e le modalità con le quali si sono manifestati questi processi. La scelta di Forza Italia è dovuta al fatto che, per la sua stessa apparizione, ha significativamente rappresentato la crisi del sistema partitico e quella più generale della democrazia italiana.

Per quanto riguarda la decisione di focalizzare l'analisi sugli amministratori locali, questa è suggerita, oltre che dalla scarsità di studi empirici sulla classe politica locale emersa nel corso della lunga transizione italiana, anche dalle evidenti difficoltà incontrate dal movimento ad approdare ad una compiuta istituzionalizzazione e, in particolare, ad affrancarsi da quel carattere «patrimoniale» o di «partito azienda» che ne ha segnato gli esordi (Maraffi 1995). La lentezza e l'ambiguità dei processi di articolazione dell'apparato interno e la conseguente carenza di strutture periferiche forti e legittimate dal basso hanno favorito, nel periodo 1995-1997, una crescita del ruolo degli amministratori locali che, al contrario della dirigenza periferica e soprattutto dei club, risultano altamente visibili in quanto stabilmente inseriti nei circuiti della rappresentanza e del governo locale, a stretto contatto con la base elettorale e da questa direttamente legittimati. Queste élites si sono affermate come elemento costitutivo di Forza Italia e assieme agli altri tre già emersi nella fase genetica - struttura centrale, gruppi parlamentari, club (Maraffi 1996) - e formano un compiuto quadro organizzativo del movimento. Pur non potendo identificare in toto il partito con i suoi eletti, si può quindi affermare che gli eletti costituiscono a tutt'oggi un elemento indispensabile della sua ossatura, che riesce ad assolvere alle funzioni di raccolta e di trasmissione della domanda politica meglio della struttura organizzativa primaria (club e gruppi dirigenti).

Una radiografia del personale eletto nei governi locali, in altri termini, può fornire un quadro di Forza Italia e dei suoi gruppi di riferimento economici e sociali forse diverso da quello emerso a livello nazionale, ma proprio per questo necessario per la piena conoscenza della nuova formazione politica. La connessione dei due livelli di analisi risulta vieppiù necessaria per almeno due motivi. In primo luogo perché la partecipazione al governo locale costituisce un requisito essenziale per il consolidamento e l'istituzionalizzazione delle organizzazioni partitiche nel nostro paese; in secondo luogo perché i rapporti 
intercorrenti fra centro e periferia hanno sempre fortemente condizionato la natura dei partiti italiani e le loro prospettive evolutive. Le informazioni sugli eletti nelle amministrazioni locali forniscono dunque elementi di grande utilità per comprendere la natura del partito come corpo agente all'interno della società civile. A maggior ragione ciò è vero nel caso di un partito che, come Forza Italia, ripudia qualsiasi forma di burocratizzazione interna e opta per modelli organizzativi atipici, ancorché dai contorni indefiniti, di volta in volta evocati da formule quali «partito leggero» o «partito americano».

La nostra analisi si muoverà quindi in due direzioni: la messa a fuoco dei tratti caratteristici di una frazione importante della nuova classe politica locale e la raccolta di elementi empirici utili a meglio comprendere la natura di Forza Italia, il suo rapporto con la società, le dinamiche interne e le sue possibili linee evolutive. In attesa di una più ampia sistematizzazione, faremo uso, in questa sede, di una parte dei dati rilevati per chiarire alcuni quesiti circa la natura attuale del movimento: modalità di selezione degli amministratori locali; caratteristiche sociali e politiche di tali amministratori; l'offerta di rappresentanza nei confronti dei vari strati sociali.

L'articolo si compone quindi di quattro paragrafi in ciascuno dei quali viene sviluppato uno degli argomenti sopra enunciati adottando, per quanto possibile, un orientamento comparato $^{2}$. Il primo paragrafo contiene una disamina dei tratti socioanagrafici degli eletti (età, sesso, titolo di studio), con riferimento alle precedenti ricerche sulla classe politica e alle teorie classiche sulla partecipazione. Il secondo paragrafo analizza gli elementi di continuità ovvero di rottura col sistema partitico preesistente, prendendo in conto esperienze partitiche e cariche partitiche e amministrative precedenti. Il terzo paragrafo, in connessione col secondo, sviluppa il tema delle modalità di reclutamento della nuova leva di amministratori locali, tenendo presente sia il ruolo svolto dalla struttura partitica che dell'apporto di altre agenzie di socializzazione politica quali i gruppi di inte-

2 Le basi di comparazione sono costituite dalle precedenti ricerche sulla classe politica locale e da quelle sui candidati e sugli eletti al Parlamento nazionale in occasione delle elezioni politiche del 1994 e del 1996. La comparazione è spuria, trattandosi di ricerche prevalentemente orientate sui consiglieri comunali; cionondimeno, anche articolando l'analisi del nostro campione per tipo di carica, le principali tendenze osservate non cambiano di molto. 
resse economico e le associazioni volontarie. Nel quarto paragrafo viene infine affrontato il tema dell'offerta di rappresentanza avanzata dagli eletti di Forza Italia nei confronti dei vari settori economici e sociali.

Anomalie e continuità del retroterra socio-anagrafico degli amministratori

La distribuzione del campione per le tre variabili socio-demografiche fondamentali - sesso, età, titolo di studio - risulta fortemente squilibrata rispetto alla popolazione italiana. Il dato, tutt'altro che inatteso, stupisce tuttavia per la sua nettezza. Come è stato evidenziato fin dalle prime ricerche sulla classe politica municipale, la rappresentatività socio-anagrafica degli eletti nelle amministrazioni locali italiane obbediva ad alcune «leggi» generali, che valevano a prescindere dal partito di appartenenza. In particolare, per quanto riguarda sesso, età e livello di istruzione, si rilevava la costante penalizzazione delle classi anagrafiche marginali (anziani e giovanissimi) rispetto a quelle centrali; delle donne rispetto agli uomini; dei meno istruiti a vantaggio degli scolarizzati, secondo una tendenza che, pur trainata dalla parallela evoluzione della società, era stata particolarmente premiante nei confronti di laureati e diplomati (Barberis 1988).

Il quadro fornito dagli amministratori locali di Forza Italia a metà degli anni Novanta non si distacca da queste direttrici di fondo, anzi le sviluppa con particolare intensità. Analizzando partitamente ciascuna variabile, osserviamo che la distribuzione per età del campione è fortemente spostata a favore delle fasce centrali, con un'età media di 44 anni (contro i 45 anni del totale degli amministratori locali) ed una classe modale, quella fra i 31 ed i 45 anni, nella quale si concentra oltre la metà dei casi (tab. 1). Gli eletti risultano così fortemente concentrati in un breve spazio anagrafico, a testimonianza di una specializzazione assai sviluppata.

Analoga difformità registriamo comparando i risultati della nostra indagine con quelli riportati nelle principali monografie sulla classe politica locale negli anni Ottanta. Per quanto riguarda la stratificazione per età osserviamo, in particolare, una netta regressione della presenza delle classi marginali (eletti di età inferiore ai 31 anni e superiore ai 60$)$ del nostro campione $(10 \%$ 
TAB. 1. Distribuzione per classe di età del campione di eletti di Forza Italia e degli amministratori locali italiani (valori percentuali)

\begin{tabular}{lcc}
\hline & Amministratori locali italiani & Eletti Forza Italia \\
\hline Da 18 a 30 anni & 7,7 & 4,4 \\
Da 31 a 45 anni & 45,1 & 54,2 \\
Da 46 a 60 & 40,6 & 35,8 \\
Oltre i 61 & 6,6 & 5,6 \\
Totale & 100 & 100 \\
& $(\mathrm{~N}=16.501)$ & $(\mathrm{N}=160)$ \\
\hline
\end{tabular}

Nota: I valori della colonna «Amministratori locali italiani» si riferiscono al totale dei consiglieri regionali, dei consiglieri comunali dei centri al di sopra dei 15.000 abitanti e dei capigruppo provinciali di tutti i partiti.

Fonte: Anagrafe degli amministratori locali del Ministero degli Interni; ricerca Cnr, Partiti e sistemi di partito in Italia. Le trasformazioni organizzative.

del totale) rispetto alla quota, già piccola, degli anni Ottanta, che si attestava costantemente attorno al $20 \%$ (Bettin e Magnier 1989). Tale processo che, come si evince dalla tabella, coinvolge tutte le forze politiche, è tuttavia meno intenso a livello aggregato dal momento che, sul totale degli amministratori locali, gli ultrasessantenni ed i meno che trentenni sono il $14,3 \%$. Possiamo quindi ipotizzare, dal momento che gli eletti di FI sono presenti in entrambi i gruppi, un comportamento ancor più favorevole a questi segmenti anagrafici da parte degli altri partiti. La differenza più chiara fra i due gruppi di amministratori è costituita dal diverso peso delle due classi centrali che vede, per FI, una netta supremazia degli eletti compresi fra i 31 ed i 45 anni, mentre a livello aggregato la classe modale è quella successiva (46-60 anni). Il dato può essere accolto, sia pure con molte cautele, come indicatore della relativa «novità» di FI rispetto agli altri partiti.

La distribuzione per sesso risulta assai squilibrata in favore degli uomini, con appena l' $8,8 \%$ di donne fra gli eletti di FI a fronte del $13,4 \%$ rilevato nell'universo degli amministratori locali. La scarsità di personale femminile deriva, oltre che dal minore tasso di successo di queste candidature, anche dall'incapacità, più volte osservata, delle formazioni di centro-destra di attrarre e sostenere con successo candidature femminili (Mattina e Tonarelli 1997; Verzichelli 1997). Minori differenze si riscon- 
TAB. 2. Distribuzione per titolo di studio del campione di eletti di Forza Italia e degli amministratori locali italiani (valori percentuali)

\begin{tabular}{lcc}
\hline & Amministratori locali italiani & Eletti Forza Italia \\
\hline Laurea & 43,3 & 66,0 \\
Diploma & 39,1 & 30,3 \\
Licenza media & 15,8 & 3,1 \\
Licenza elementare e senza titolo & 1,8 & 0,6 \\
Totale & 100 & 100 \\
\hline
\end{tabular}

Fonte: Si veda la tabella 1.

trano nella comparazione con i risultati di altre ricerche (Bettin e Magnier 1989; Barberis 1988). In questo caso possiamo affermare che si replica un copione già noto: la quota di donne elette in Forza Italia risulta infatti sempre molto superiore a quella di elette nelle file del pentapartito e inferiore a quella delle elette nel Pci degli anni Ottanta. Il dato sulla presenza femminile è sostanzialmente in linea con quello registrato a livello dei gruppi parlamentari: $10,3 \%$ alla Camera e $2,1 \%$ al Senato. In ogni caso, l'affermazione di FI non implica significative aperture d'accesso a vantaggio della popolazione femminile e, anzi, rispetto alle altre forze politiche si registra un cospicuo arretramento (5,6 punti percentuali) da posizioni invero già precarie.

Una maggiore e ulteriore espressione della peculiarità degli eletti di Forza Italia si riscontra esaminando il loro livello di istruzione, indicatore, allo stesso tempo, di estrazione sociale e, almeno in potenza, di capacità politica. I titoli di studio di cui sono in possesso gli eletti del campione sono per la maggior parte medio-alti $(66 \%$ di laureati, a cui si contrappone l'esiguo $3,6 \%$ degli eletti che non sono andati oltre la scuola dell'obbligo).

L'elevatissima quota di laureati è il dato in assoluto più interessante che ci consegna la ricognizione sociografica. Tale dato è infatti il più divergente non solo rispetto alla popolazione italiana in generale, nella quale i laureati sono il 3,6\%, ma anche rispetto agli amministratori locali del recente passato, fra i quali la percentuale di laureati non è mai stata superiore al 25\% (Barberis 1988; Bettin e Magnier 1989). Come si vede confrontando le due colonne della tabella 2, la tendenza alla crescita dei laureati fra gli eletti non riguarda solamente FI ma, 
in generale, tutti i partiti $(43,3 \%)$ ed è un fenomeno che si manifesta a livello di tutte le assemblee elettive. Forza Italia, tuttavia, è il partito che mantiene sempre la maggior quota di laureati fra i propri eletti. Degno di nota è anche il fatto che la quota di laureati del nostro campione sia di poco inferiore a quella che si registra all'interno del gruppo parlamentare di FI della Camera dei deputati $(73,5 \%)$, segno evidente di un tratto generale caratteristico e qualificante (Verzichelli 1997).

$\mathrm{Al}$ termine di questo paragrafo possiamo avanzare alcune prime conclusioni. Innanzitutto, osserviamo come, rispetto alle tre variabili di base (sesso, età, titolo di istruzione), il gruppo degli amministratori locali di Forza Italia si presenti sostanzialmente in linea con i gruppi parlamentari e con i delegati al congresso di Milano dell'aprile $1998^{3}$. In secondo luogo, si rileva che la «sindrome dis-rappresentativa» manifestata dai partiti politici italiani nel periodo antecedente la nascita di Forza Italia, e caratterizzata dalla sistematica associazione di tre deficit (classi anagrafiche marginali, presenza femminile, bassi livelli di istruzione), viene pienamente condivisa dal nuovo movimento che addirittura la sviluppa in forma accentuata. Se il ridotto peso dei titoli di studio inferiori ha senz'altro carattere fisiologico in un quadro di generale crescita dei livelli di istruzione, $\mathrm{i}$ deficit relativi alla presenza femminile e delle classi anagrafiche marginali, e in particolare dei più giovani, sono più significativi, in quanto attestano la minore capacità del nuovo partito di sollecitare l'attivismo politico di segmenti sociali rilevanti.

Una prima lettura in chiave comparata di questi dati indica una certa peculiarità degli eletti di Forza Italia, sia nei riguardi della popolazione italiana nel suo complesso che della classe politica degli anni Ottanta. Tutto ciò si può riassumere col concetto di sovrarappresentazione dei segmenti centrali, ossia di quegli strati della popolazione maggiormente dotati di risorse sociali primarie: un'età mediana a cui si associa normalmente il pieno inserimento nel mondo del lavoro e delle relazioni sociali; il sesso maschile; un titolo di studio elevato con tutte le opportunità in termini di prestigio, carriera professionale e visibilità sociale ad esso collegate.

3 Cfr. «la Repubblica», 15 aprile 1998. 


\section{Le leve del potere locale fra Prima e Seconda repubblica}

Data l'indiscutibile novità di Forza Italia nel quadro politico italiano, c'è da chiedersi se essa sia rilevabile anche nelle modalità di reclutamento delle sue élites locali e nel loro grado di professionalizzazione politica. Abbiamo perciò cercato di controllare se il pattern di reclutamento deducibile dall'analisi dei dati sia confrontabile con quelli già esistenti e adottati da altri partiti e da Forza Italia stessa per la selezione dei gruppi parlamentari. Attraverso tale ricognizione si è tentato di controllare l'esistenza di un precedente percorso politico degli eletti ed il loro coinvolgimento nella direzione dei partiti e/o nel governo della cosa pubblica prima della nascita del movimento. Ciò dovrebbe consentirci di desumere la presenza di personale già professionalizzato e l'area politica di provenienza. Dovremmo, di conseguenza, poter chiarire se, tramite l'elezione nelle liste di Forza Italia, si sia affermata una leva del tutto nuova di politici locali o, al contrario, vi sia stato solo un reimpiego di personale politico già socializzato da altre formazioni politiche.

I dati raccolti configurano un quadro composito, articolato su due distinti modelli di selezione; un quadro imputabile ai comportamenti della dirigenza e al libero gioco delle forze sociali secondo combinazioni che esamineremo nel prossimo paragrafo. L'esame del primo indicatore impiegato, la precedente iscrizione a partiti politici, evidenzia l'esistenza di una rilevante quota di eletti $(56,2 \%)$ che in passato aveva aderito ad altre formazioni politiche, mentre solo il $43,8 \%$ dichiara di essere privo di precedenti affiliazioni (tab. 3). Fra i partiti spiccano la Dc ed il Psi che da soli danno conto del 67,8\% delle precedenti affiliazioni e del $38,1 \%$ dei casi, mentre complessivamente gli eletti provenienti dall'area dell'ex pentapartito giungono a rappresentare l' $^{\prime} 88,9 \%$ dei già iscritti ed il $50 \%$ dei casi del campione.

Da quest'angolo visuale è indubbio che gli eletti si pongono in linea di diretta continuità con le élites politiche della Prima repubblica. In particolare, si caratterizzano per una fortissima specializzazione governativa a cui corrisponde una modesta presa del Movimento sociale e la totale assenza di precedenti politici nelle opposizioni di sinistra. La passata iscrizione risulta, come atteso, correlata positivamente all'età. Ciò nonostante, anche fra le classi più giovani la quota dei già iscritti si mantiene sempre al di sopra del $50 \%$, a testimoniare l'esistenza di una 
TAB. 3. Precedenti affiliazioni partitiche degli eletti di Forza Italia

\begin{tabular}{lcc}
\hline & \% sul totale & \% sui già iscritti \\
\hline Dc & 25,6 & 45,6 \\
Psi & 12,5 & 22,2 \\
Pli & 5,0 & 8,9 \\
Pri & 3,8 & 6,7 \\
Psdi & 1,9 & 3,3 \\
Dc e laici & 1,2 & 2,2 \\
Msi & 3,1 & 5,6 \\
Lega Nord & 1,3 & 2,2 \\
Ppi & 0,6 & 1,1 \\
Patto Segni & 0,6 & 1,1 \\
An & 0,6 & 1,1 \\
Non iscritti in precedenza & 43,8 & - \\
& & \\
Totale & 100 & 100 \\
& $(\mathrm{~N}=160)$ & $(\mathrm{N}=90)$ \\
\hline
\end{tabular}

professionalità politica, sia pure incipiente, già sviluppata in precedenza e verosimilmente sensibile alle opportunità fornite dalla lunga transizione italiana (Recchi 1989).

Indubbiamente le differenze con gli eletti al Parlamento e con la declamata novità del partito sono forti. Tuttavia è opportuno affinare ulteriormente l'analisi. Posta l'esistenza di un folto gruppo di eletti con precedenti esperienze partitiche, quanto esse possono avere inciso sulla formazione politica, sul know how e sulla vocazione rappresentativa degli eletti? A questo proposito è utile verificare il tipo di incarichi ricoperti in seno ai partiti, la quota di eletti che hanno già alle spalle anche incarichi elettivi in amministrazioni pubbliche, nonché l'importanza di tali cariche. I dati relativi allo status raggiunto in seno alle precedenti formazioni partitiche sono, tuttavia, meno chiari. In primo luogo, ciò è dovuto a una rilevante quota di non risposte, che inficia l'affidabilità dei risultati per le domande in questione. I dati raccolti consentono, comunque, di ipotizzare che si sia trattato di personale politico dallo status relativamente basso in ragione della quota relativamente modesta dei già iscritti che affermano di aver ricoperto cariche partitiche di rilievo $(26,9 \%)$. Similmente, i riscontri empirici relativi alle precedenti esperienze amministrative attestano complessivamente il basso rango degli eletti. La quota di coloro che hanno ricoperto cariche politico-amministrative è infatti piuttosto bassa $(34,4 \%)$ e fra di essi prevalgono gli incarichi di basso livello. Tuttavia, accorpando in 
un'unica categoria coloro che hanno avuto esperienze direttive in seno a partiti politici e/o nel governo locale, definendo cioè una categoria di politici per lo meno semi-professionalizzati, si raggiunge il $43,1 \%$ del campione, una quota considerevole che merita senz'altro un supplemento di riflessione.

E fuor di dubbio che $\mathrm{i}$ dati raccolti suonano in netto contrasto con le caratteristiche del personale parlamentare di FI e con le principali opzioni di valore del movimento. Se un profondo ricambio della classe politica era considerato uno degli obiettivi primari del programma di Forza Italia, si deve concludere che le logiche affermatesi in periferia hanno obbedito ad altri imperativi e hanno relegato in secondo piano gli eventuali impulsi della struttura centrale. Se, da un lato, vi è l'esigenza di un profondo rinnovamento della classe dirigente, questo non può realizzarsi appieno e su tutti i fronti e, soprattutto, non a livello locale, dove non sembra possibile reperire candidati dotati di risorse politiche rilevanti al di fuori dei partiti tradizionali. In periferia, peraltro, si registra la disponibilità di una classe politica già esperta, di matrice prevalentemente pentapartitica e ancora capace di connettersi al vasto serbatoio del voto moderato, che mette a disposizione del nuovo movimento la propria expertise politica ed il proprio radicamento nella comunità locale. Merita dire inoltre che, a seguito del terremoto politico del 1994, questa classe politica superstite risulta in soprannumero rispetto alla forza residua dei partiti originari o di quelli da essi immediatamente derivati, e per tale ragione si presta ben volentieri a colmare i vuoti di organico lamentati, a livello locale, dai nuovi partiti e da Forza Italia in particolare (Maraffi 1996).

Il livello locale è dunque il luogo in cui l'opera di rinnovamento rallenta la sua corsa sia a causa della residua forza degli apparati partitici sia, soprattutto, a causa della ancor debole ramificazione del nuovo movimento e dalla carenza di quadri che ne consegue. Il quadro che ne risulta si caratterizza, quindi, per la sovrapposizione e la contemporaneità di due modelli antitetici, ma funzionalmente complementari, di selezione del personale politico. Il primo modello, centrato sulla società civile e sulla estraneità alla politica, si è rivelato particolarmente efficace per reclutare il personale politico parlamentare. Il secondo si fonda invece sul reimpiego di spezzoni della precedente classe politica. Con questo secondo modello il nuovo partito ha fatto fronte al problema della costituzione di un ceto politico locale altrimenti non reclutabile nel breve lasso di tempo inter- 
corso fra la sua nascita e le amministrative del 1995. La contiguità degli eletti di FI a livello locale con il ceto politico dei partiti della Prima repubblica è, dunque, netta. Tuttavia, più che a una semplice riproposizione delle vecchie élites locali, ci si trova di fronte a una crescita del ruolo delle seconde file del pentapartito.

\section{Modalità di reclutamento}

Verificata l'esistenza del carattere composito della classe politica locale in Forza Italia, è utile ora domandarci secondo quali modalità e secondo quali criteri si sia sviluppato il processo di selezione delle candidature, con particolare riferimento al ruolo svolto dalle strutture periferiche del movimento. Gli elementi che sono stati presi in considerazione per mettere in luce i percorsi di accesso alla politica alternativi e concorrenti rispetto alla militanza pentapartitica, riguardano la famiglia, le associazioni e la struttura organizzativa di FI.

Rispetto al ruolo giocato dalla famiglia, osserviamo che questo è. di notevole rilievo dal momento che, sommando $i$ casi in cui almeno uno dei genitori degli eletti è stato iscritto ad un partito politico o ha ricoperto un incarico elettivo, costituiscono il $41,3 \%$ del totale. Ciò significa che, come evidenziato in altre ricerche (Recchi 1989), i nostri eletti si trovavano, già da prima, relativamente vicini alla politica. A questo proposito non si può fare a meno di segnalare una evidente contiguità con gli orientamenti politici dei genitori, fra i quali l'area pentapartitica risulta dominante, e con le opzioni politiche manifestate in gioventù attraverso la militanza in associazioni studentesche di prevalente ispirazione laico-socialista e cattolica.

Per quanto riguarda la carriera all'interno di Forza Italia, osserviamo che il $45 \%$ degli eletti ricopre tuttora cariche all'interno del partito. Sommando, inoltre, coloro che ricoprono attualmente cariche nel partito con quanti ne hanno detenute in passato, otteniamo il $62,5 \%$. Quindi la carriera all'interno di Forza Italia, per breve che possa essere stata, costituisce un fattore determinante per il successivo accesso alle cariche elettive. Ma l'impressione che il partito possa avere avuto un ruolo esclusivo e/o determinante nel regolare gli accessi alle istituzioni elettive deve essere corretta alla luce dei dati relativi alle concrete modalità di definizione delle candidature. Notiamo infatti 
TAB. 4. «Come potrebbe descrivere l'appoggio ricevuto in campagna elettorale da...?» $(\max =3 ; \min =0)$

Fornitore di sostegno

Da parte di alcuni amici

2,66

Dal comitato elettorale

1,80

Dal mio partito

1,56

Dai club di Forza Italia

1,43

$\mathrm{Da}$ categorie professionali/sindacati

1,35

Dal coordinatore regionale di Forza Italia

1,05

Dalla coalizione dei partiti

0,98

Da associazioni cattoliche

0,94

Dai giornalisti locali

0,91

che l'elemento partitico non è né esclusivo né preclusivo nei confronti di altre forme di accesso all'agone politico, dal momento che il $33,8 \%$ degli intervistati afferma di aver proposto di propria iniziativa la candidatura e che il $20 \%$ indica in «amici attivi nel movimento» il principale punto di contatto per la proposizione della candidatura piuttosto che la struttura organizzativa locale. Osserviamo inoltre che ben il $91,9 \%$ degli eletti sostiene di non aver ricevuto alcun sostegno economico dal partito a fronte di un $56,9 \%$ che afferma di aver provveduto per intero alle spese della campagna elettorale. Analoghe considerazioni si possono fare per l'appoggio ricevuto in campagna elettorale, nel corso della quale le principali basi di sostegno sono state gli amici e il comitato elettorale, mentre il partito è solo terzo (tab. 4).

Dal quadro così delineato si desume che la struttura partitica ha giocato un ruolo sostanzialmente formale e tutto sommato marginale, sia nei processi di selezione delle candidature che nella successiva fase di promozione delle stesse. Sembra quasi che il partito, una volta messo a disposizione un marchio elettorale, abbia lasciato aperti ampi varchi al protagonismo dei singoli e, in particolare, di coloro che si sono mostrati capaci di mobilitare risorse di tipo societario. L'ambito principale in cui maturano tali risorse è quello associativo e delle relazioni amicali. I componenti del nostro campione, anche in ragione della particolare estrazione professionale (si veda oltre), risultano infatti particolarmente inclini ad aderire ad associazioni sia di rappresentanza di interessi economici che volontarie e, in conseguenza di ciò, a coltivare una estesa rete di contatti e relazioni personali che ne aumentano il credito e la visibilità sociale. 
TAB. 5. Adesione ad associazioni non economiche da parte degli eletti di Forza Italia (valori percentuali sul totale delle associazioni citate)

\begin{tabular}{lc}
\hline Associazioni sportive & 32,8 \\
Lions club, Rotary e simili & 26,2 \\
Associazioni culturali & 12,3 \\
Associazioni di volontariato & 10,7 \\
Associazioni cattoliche & 4,9 \\
Associazioni ambientaliste & 3,3 \\
Altre & 9,8 \\
Totale & 100 \\
& $(\mathrm{~N}=194)$
\end{tabular}

I dati raccolti ci consentono di sviluppare il tema della centralità sociale partendo dalla propensione associativa, che della centralità costituisce un indicatore essenziale. Partendo dall'associazionismo economico, osserviamo che coloro che risultano attualmente iscritti, o che sono stati iscritti in passato ad associazioni di rappresentanza degli interessi, sono il $44,4 \%$ del campione, e che non piccola è la quota di coloro che vi hanno ricoperto ruoli direttivi $(28,1 \%)$. Evitando per il momento di esaminare le singole categorie di associazioni citate, assumiamo questo dato come indicatore di un percorso di accesso alla politica che concorre, assieme $o$ in competizione col canale partitico, alla produzione di personale politicamente esperto e socialmente visibile. Per quanto riguarda le associazioni volontarie a carattere non economico, osserviamo che ben il $55 \%$ degli eletti aderisce ad almeno una associazione di questo tipo, per una cifra complessiva di 194 adesioni, pari ad una media di 2,2 adesioni procapite. La tabella 5 dà conto dell'incidenza di ogni singola famiglia associativa sul totale.

Come già è stato rilevato per i candidati al Parlamento nazionale (Mattina e Tonarelli 1997), il personale politico di Forza Italia risulta fortemente partecipe delle attività di associazioni volontarie, indicando in tal modo una posizione sociale caratterizzata dal pieno inserimento nella collettività già in precedenza ed a prescindere dall'elezione. In particolare, la forte propensione associativa si indirizza verso due gruppi distinti di associazioni, quelle sportive e quelle a carattere notabiliare (Lions club, Rotary e altre), che si qualificano per essere luoghi privilegiati di incontro tra élites e punti di riferimento di ampi strati della società: in una parola, che manifestano una chiara potenzialità politica. 
In termini generali, possiamo dire che il ruolo delle associazioni, economiche e non, si configura come sostitutivo/integrativo nei confronti del canale partitico, che abbiamo già rilevato come insufficiente a dare conto dei processi di selezione. Diversi studi hanno messo in evidenza la forte correlazione esistente tra la partecipazione associativa e il livello del coinvolgimento individuale nella sfera politica. Già a partire dagli anni Sessanta, una consolidata letteratura di matrice anglosassone, fondata su ricerche empiriche, ha individuato una serie di correlazioni positive molto accentuate fra adesione ad associazioni, coinvolgimento psicologico nella politica e partecipazione politica, sia per ciò che attiene il comportamento elettorale sia per ciò che riguarda la socializzazione del personale elettivo (Key 1960; Almond e Verba 1963; Verba e Nie 1972). Ne consegue che l'adesione a, e la direzione $\mathrm{di}$, associazioni volontarie, anche $\mathrm{di}$ quelle non a carattere politico, costituisce un importante «predittore» dell'attività politica dei cittadini ${ }^{4}$.

Nell'attuale contesto italiano, caratterizzato dalla crisi dei partiti di massa, si può ravvisare nella partecipazione associativa un momento di un processo di modernizzazione che, rompendo con modalità di militanza dai caratteri totalizzanti, offre una moltiplicazione delle occasioni di partecipazione che allargano le potenzialità di formazione e selezione del personale politico, fornendo così un serbatoio anche per i processi di ricambio del ceto politico (Ramella 1994). Ma, al di là delle fasi, pur centrali, della partecipazione e della mobilitazione, il canale associativo si rivela prezioso anche per la messa a punto di un know bow politico vero e proprio. Le esperienze associative, infatti, sono spesso occasione per un tirocinio formativo che dà luogo allo sviluppo di competenze organizzative e di socializzazione del ceto politico; sul piano locale, inoltre, la notorietà acquisita dai leader associativi conferisce loro una risorsa in più per competere con successo nell'arena politica (Sills 1972; Bequart Leclercq $1976)^{5}$. Se ciò è vero in termini generali, lo è ancor di più nel caso di Forza Italia, ove si riscontrano la rinuncia $a b$ initio a qualsiasi richiamo al partito di massa e una articolazione organizzativa ancora rudimentale, specie a livello locale. Il canale partitico, dunque, lungi dall'annullarsi, viene ad essere affianca-

4 Sul punto vedi l'ampia trattazione svolta da Ramella (1994).

5 Per il caso italiano vedi in particolare Diamanti (1995). 
to ed integrato da una serie di altri vettori di politicizzazione tutti facenti capo, in modo più o meno diretto, ad una posizione sociale privilegiata ed alla rete di rapporti e scambi che ad essa corrisponde.

In conclusione, possiamo affermare che, a livello locale, il reclutamento degli amministratori di Forza Italia si è sviluppato facendo leva su sollecitazioni di differente natura, nel contesto di un disegno organizzativo che è riuscito, almeno per il momento, ad integrare percorsi funzionalmente complementari, ma potenzialmente contraddittori. Da un lato, si è manifestata l'esigenza di mobilitare gruppi nuovi a forte visibilità sociale e portatori di competenze professionali pregiate, capaci di supplire alla carenza di expertise politica. Dall'altro, ci si è affidati alla disponibilità di spezzoni rilevanti del vecchio ceto politico-amministrativo a mettere al servizio di Forza Italia il proprio know how e la propria rete di rapporti. Ne è risultato un modello di reclutamento promiscuo nel quale gli elementi innovativi convivono con forti elementi di continuità col passato. Nel complesso, i percorsi di accesso disegnati dai dati raccolti attestano, a livello globale, la prevalenza dei tratti tipici di un modello di reclutamento a carattere societario (Verzichelli 1997), mentre i numerosi elementi di continuità con le formazioni politiche della Prima repubblica indicano l'esistenza di una comune area politica e sociale di riferimento piuttosto che la duplicazione di metodi di selezione già sperimentati. Nel nostro caso, il partito resta sullo sfondo o, per meglio dire, limita il suo ruolo a quello di regolatore, più o meno zelante, degli accessi a fronte di una autonoma attivazione di soggetti «altri», fra i quali dominano le precedenti formazioni politiche e ampi settori della società civile. Gli interrogativi che si pongono per il futuro riguardano la natura transitoria o permanente di questa situazione e l'eventuale affermazione di un ruolo partitico più incisivo. In ogni caso, la direzione nella quale evolverà il reclutamento degli amministratori locali di Forza Italia, e l'eventualità di una cristallizzazione attorno a un modello definito, dipenderanno in buona misura dal ruolo che il partito potrà/vorrà ricoprire nella rappresentanza degli interessi sociali e dalla natura di questi ultimi. 


\section{L'offerta di Forza Italia}

Resta, allora, da chiarire di quali interessi Forza Italia sia portatrice nelle istituzioni del governo locale e, più in generale, quali siano le istanze, espresse e latenti, che riescono a trovare il maggiore sostegno da parte dei suoi rappresentanti. Rispondere a questo interrogativo è operazione complessa e non priva di rischi metodologici. Tuttavia, l'impianto del questionario ci consente di dare almeno una parziale risposta partendo da tre indicatori fondamentali: a) la composizione sociale degli eletti; $b$ ) la diretta presenza di associazioni economiche; $c$ ) la mission rappresentativa che gli eletti si auto-attribuiscono, cioè le auto-rappresentazioni del ruolo politico. I primi due indicatori attengono alla sfera della rappresentatività e riguardano la rappresentanza politica solo in modo indiretto; il terzo invece ha carattere marcatamente politico e sarà trattato nel prossimo paragrafo.

Il primo indicatore proposto, la professione degli eletti, si affianca a quelli già esaminati del sesso, dell'età e del titolo di studio. Assieme ad essi, ma su un piano che abbiamo voluto tenere distinto, ci consente di stimare l'offerta di rappresentanza sub-specie di rappresentatività sociologica del corpo politico relativamente ai cruciali aspetti dell'appartenenza di classe e della discriminante fra settore pubblico e privato (Cotta 1986). Esso, inoltre, ci permette di controllare se, grazie a Forza Italia, stiano entrando nell'arena politica nuove figure professionali specializzate nella rappresentanza, capaci di sostituire il monopolio storicamente assunto dal funzionariato pubblico e partitico (Barberis 1988). Si tratta allora di considerare, in via preliminare, se e quanto l'estrazione sociale degli eletti rispecchi determinati raggruppamenti sociali ovvero tenda ad appiattirsi su quella che è la struttura generale della popolazione.

Naturalmente sarebbe errato identificare pedissequamente la rappresentanza sociologica con quella politica. La concezione della rappresentanza come semplice specchio, vale a dire l'ipotesi che $\mathrm{i}$ rappresentanti sarebbero in perfetta sintonia con $\mathrm{i}$ rappresentati per il solo fatto di essere loro sociologicamente affini, è semplicistica al punto di ridurre la figura del politico ad un ruolo passivo che cozza con le più elementari acquisizioni del senso comune. Sul piano interpretativo, tuttavia, non è inutile riflettere sulla composizione sociologica degli eletti, dal momento che le motivazioni all'azione politica, sia in termini psicologici che di difesa degli interessi dei diversi settori sociali, 
non possono scindersi del tutto dalle appartenenze sociali originarie (Bettin e Magnier 1989). In altre parole, possiamo assumere che la contrapposizione degli interessi sociali emerge anche dalla composizione sociale delle élites politiche (Barberis 1988). La rappresentanza sociologica degli eletti costituisce così un valido, anche se imperfetto, indicatore della rappresentanza politica; specularmente, la composizione socio-professionale del campione fornisce anche un'informazione circa i ceti sociali maggiormente disponibili al diretto impegno in politica e più sensibili al richiamo di Forza Italia a prescindere dal tipo di interessi che essi finiranno per rappresentare nell'arena politicoamministrativa.

A questo proposito i dati della tabella 6 attestano una fortissima specializzazione e un livello minimo di interclassismo fra gli eletti di Forza Italia. Tre sole categorie professionali (liberi professionisti, imprenditori e dirigenti d'azienda) danno, infatti, ragione di ben il 56,3\% del campione, mentre, da una parte, è ridotta la quota di eletti fra le categorie professionali tradizionalmente prevalenti fra i partiti di massa italiani (politici di professione e insegnanti) e, dall'altra, è ancora di una certa consistenza la presenza di dipendenti pubblici. In linea generale, si nota che Forza Italia manifesta, anche a livello locale, un profilo assolutamente peculiare di partito del settore privato e delle classi abbienti, in primo luogo; in ogni caso delle classi a più forte visibilità sociale e centrali nei rapporti sociali ed economici. A livello di macro-aggregazioni, infatti, il rapporto fra eletti ascrivibili al settore pubblico e al settore privato è di 1 a 3 . In particolare, si osserva la netta affermazione di un determinato tipo di attività, quella del libero professionista - segnatamente avvocato o commercialista - in una misura tale $(37,5 \%)$ da sopravanzare di gran lunga la presenza degli imprenditori, la cui quota, peraltro, si attesta su valori assai elevati $(11,9 \%)$. Lo svolgimento di una libera professione si configura, dunque, come la condizione lavorativa tipica dei politici locali di Forza Italia, allo stesso modo di quanto avveniva per gli insegnanti e i pubblici funzionari nei grandi partiti della Prima repubblica. Ciò attesta l'esistenza, sia pure in nuce, di un modello alternativo di professionalizzazione del ceto politico in cui predominano alcune delle caratteristiche del politico di carriera (Mastropaolo 1993). Va inoltre segnalata la presenza assai modesta di magistrati e professori universitari, la quasi assenza di coltivatori diretti e la quota - assai elevata per questo genere di categorie - 
TAB. 6. Professione degli eletti (valori percentuali)

\begin{tabular}{lc}
\hline Professione degli eletti & $\%$ \\
\hline Liberi professionisti & 37,5 \\
Dipendenti pubblici & 16,9 \\
Imprenditori & 11,9 \\
Dirigenti d'azienda & 6,9 \\
Terziario privato & 5,7 \\
Politici e sindacalisti & 5,0 \\
Commercianti ed artigiani & 4,4 \\
Studenti e assimilati & 2,6 \\
Insegnanti & 2,5 \\
Docenti universitari e magistrati & 2,5 \\
Altri & 2,4 \\
Giornalisti & 1,9 \\
Totale & 100 \\
& $(\mathrm{~N}=160)$ \\
\hline
\end{tabular}

Fonte: Per la definizione di «terziario privato», si veda Mattina e Tonarelli (1997).

di artigiani ed esercenti, i quali, inoltre, sono riusciti a far eleggere una buona percentuale di leader associativi (vedi tab. 6). Degna di nota, infine, è anche la particolare composizione interna del ridotto contingente di politici e sindacalisti, che fa capo esclusivamente alle organizzazioni di categoria del lavoro autonomo, in particolare alla Confcommercio, ed è del tutto priva di sindacalisti del lavoro dipendente.

Dal punto di vista della rappresentanza sociologica, dunque, Forza Italia raccoglie a piene mani l'attivismo delle classi medio-alte con una accentuata propensione verso le libere professioni, che costituiscono la classe modale della distribuzione $(37,5 \%)$. La particolare composizione professionale degli eletti rappresenta senza dubbio la più chiara novità espressa dall'ingresso di Forza Italia nell'agone politico. La sua presenza a livello locale, infatti, inverte decisamente la tendenza, più che trentennale, all'espulsione dei ceti professionali e produttivi dalle amministrazioni locali a vantaggio del funzionariato partitico e pubblico (Barberis 1988). Nel caso di Forza Italia si assiste quindi ad un impegno diretto dei segmenti sociali e, come vedremo, dei gruppi di interesse a cui fa riferimento il partito; elemento, questo, che determina un elevato grado di omogeneità fra rappresentanza sociologica e programmi politici. In sintesi, possiamo affermare che la composizione sociale degli eletti si 
conforma in modo più che apprezzabile alla cultura politica ed agli items programmatici più ricorrenti del movimento. La quasi assenza di magistrati, lo scarso peso di politici e sindacalisti ${ }^{6}$, l'esclusione di qualsiasi categoria di non attivi (pensionati, casalinghe, disoccupati), assieme al cospicuo inserimento di imprenditori, professionisti ed attivi nel settore privato, è evidentemente frutto di un preciso intento di incarnare, nelle file degli eletti, le opzioni di valore più nette e unificanti per l'intero movimento. A tale proposito possiamo ben affermare che la rappresentanza espressa dalla composizione sociale del campione, oltre a valere di per sé quale indicatore remoto di offerta politica, configura anche un'offerta di rappresentanza di tipo simbolico ed evocativo (Cotta 1986; Mattina e Tonarelli 1997), tesa a privilegiare i valori dell'impresa e del settore privato. Quel 23,2\% di eletti reclutati fra imprenditori, dirigenti del settore privato, artigiani ed esercenti, rappresenta la percentuale più alta mai fatta registrare nell'Italia repubblicana di presenza di questi ceti sociali nell'arena politica. L'estesa presenza di liberi professionisti non contraddice questa interpretazione, sia per il fatto che essi stessi sono fra i soggetti privilegiati nel discorso politico di FI, sia perché questa categoria svolge di norma funzioni importanti di servizio all'impresa. La nostra interpretazione è che a causa delle maggiori difficoltà degli imprenditori ad un diretto impegno in politica, l'obiettivo di affermare la centralità dei valori di impresa abbia dovuto attuarsi mediante un massiccio reclutamento di liberi professionisti i quali, in mancanza di una struttura partitica ramificata e professionalizzata, costituiscono spesso gli unici depositari dell'expertise politica (in senso ampio) e della visibilità sociale necessarie per affrontare con successo un'elezione di tipo maggioritario. In questo caso si ha l'affermazione di una sorta di vincolo tecnico, che stempera ma non annulla la tendenza generale a fare del corpo vivente del partito la rappresentazione di un modello di società.

Per quanto riguarda il secondo indicatore di rappresentatività, la diretta presenza di gruppi di interesse economico, abbiamo già detto del ruolo supplente/integratore svolto dall'associazionismo nel reclutamento degli amministratori. Ci soffermeremo adesso sullo stesso punto cercando però di valutare la sua

6 La ridotta quota di politici e sindacalisti di mestiere, laddove non sia effetto di una volontà mimetica, conferma quanto già detto riguardo al basso status degli eletti con precedenti esperienze politiche. 
TAB. 7. Associazioni sindacali e di categoria alle quali gli eletti di Forza Italia sono iscritti $e$ delle quali sono o sono stati dirigenti (valori percentuali)

\begin{tabular}{lcc}
\hline & Attualmente iscritti & Dirigenti ed ex dirigenti \\
\hline Confcommercio & 6,9 & 3,8 \\
Ordini professionali & - & 6,4 \\
Sindacati autonomi & 5,6 & 6,9 \\
Confindustria & 3,1 & 0,6 \\
Cisl & 3,1 & 5,0 \\
Confagricoltura & 2,5 & 2,5 \\
Confartigianato & 1,3 & 1,9 \\
Uil & 1,3 & 1,9 \\
Confapi & 1,3 & 1,9 \\
Coldiretti & 0,6 & 0,6 \\
Cgil & 0,6 & - \\
Altre associazioni imprenditoriali & 3,1 & 3,1 \\
Non indica & 64,6 & - \\
Totale & & 28,1 \\
& 100 & $(\mathrm{~N}=160)$ \\
\hline
\end{tabular}

qualità di anticipatore del tipo di rappresentanza di cui questi si fanno portatori. Come già si è visto, il nostro campione risulta fortemente permeabile ai gruppi di interesse economico, dal momento che il $44,4 \%$ degli eletti ne fa o ne ha fatto parte e quel che più conta - la loro presenza è assai qualificata, dato che il $28,1 \%$ degli eletti afferma di ricoprire o di aver ricoperto incarichi direttivi in tali associazioni. Coloro che risultano attualmente iscritti ad associazioni di rappresentanza economica sono pari al 35,4\% del campione e su di essi centreremo l'analisi che segue valutando partitamente i principali raggruppamenti associativi rilevati (tab. 7).

Bisogna puntualizzare che questa quota, non enorme, di membri di associazioni di rappresentanza è dovuta all'elevato numero di liberi professionisti i quali, aderendo obbligatoriamente ad ordini professionali, non sono stati inclusi fra le associazioni di tabella 7 , che sono tutte a carattere volontario ${ }^{7}$. In realtà la densità dei gruppi è elevatissima, specie fra gli eletti che svolgono attività di tipo imprenditoriale e fra i lavoratori autonomi. Infatti il $18,8 \%$ di aderenti ad organizzazioni imprendito-

7 Non di meno abbiamo un elevato numero di dirigenti o ex dirigenti di ordini professionali che, sommati alla seconda colonna della tabella 7 , porta la presenza di dirigenti dei gruppi di interesse in senso ampio al $34,5 \%$ del campione. 
riali e del lavoro autonomo che troviamo nella tabella 7 (Confindustria, Confapi, Confcommercio, Confagricoltura, Confartigianato, Coldiretti e altre) rappresenta una quota molto elevata di quel $21,9 \%$ di eletti (tab. 6) che svolgono attività suscettibili di essere inquadrate dalle organizzazioni professionali citate.

Scendendo nel dettaglio, osserviamo che le appartenenze risultano fortemente polverizzate in una grande pluralità di sigle, dalla quale emerge tuttavia con chiarezza un netto discrimine professionale e di classe. Risulta a questo proposito molto evidente l'irrilevanza della quota di adesione alle tre grandi confederazioni sindacali (Cgil, Cisl, Uil), che complessivamente contano solo sul $5 \%$ di iscritti. Tale quota peraltro è imputabile per la maggior parte alla Cisl, in evidente connessione con l'estrazione democristiana di molti degli eletti. Molto significativa è, invece, la presenza delle associazioni imprenditoriali che, complessivamente intese, coinvolgono il $18,8 \%$ del campione. Il peso della Confindustria rimane tuttavia limitato $(3,1 \%$ di iscritti fra gli eletti), mentre è molto superiore quello delle organizzazioni minori, che rappresentano i settori economici tradizionali, più esposti all'intensificarsi della concorrenza e maggiormente mobilitate sui temi della fiscalità: a Confcommercio, Confartigianato e Confapi e alle varie associazioni agricole aderisce infatti il $12,6 \%$ degli eletti.

Il rilevante ingresso dei gruppi di interesse economico nell'agone politico locale è una ulteriore attestazione della fase di transizione del sistema partitico e, in particolare, della precarietà e della fluidità dei rapporti fra i gruppi stessi e i partiti. Ma tale ingresso, nel caso qui in esame, si deve a quelle associazioni imprenditoriali minori che, all'interno di un sistema associativo imprenditoriale debole, frammentato e fortemente condizionato dalle logiche del collateralismo politico, si sono storicamente schierate con la Dc, costituendone uno dei principali interlocutori funzionali. In particolare, come segnalato in un recente studio (Ferrante 1998), tutte le associazioni di rappresentanza della piccola impresa, del commercio, dell'artigianato e dell'agricoltura, si trovano oggi ad affrontare una vertiginosa crescita della domanda di rappresentanza causata dalla liberalizzazione dei mercati, dall'intensificarsi della concorrenza e dai progetti di riforma fiscale.

Nel caso di quelle associazioni che in passato avevano costituito, per ciascun settore, il braccio sindacale della Dc, a tali sfide se ne aggiunge un'altra, quella della dissoluzione del referen- 
te partitico e della diaspora dei suoi eredi in schieramenti concorrenti. Tutto ciò fa da propellente a processi interni a ciascun singolo settore economico, che spingono verso una progressiva perdita di autonomia - di cui la partecipazione alle contese elettorali è sintomo - nonostante la tradizionale vocazione governativa di questo tipo di attori e i noti rischi corsi, in contesti politici tendenzialmente maggioritari, dai gruppi troppo esposti (Mattina 1994). Nel caso delle associazioni vicine alla sinistra, questa tendenza viene attenuata dalla maggiore solidità dei referenti politici e, a partire dal 1995, dal loro stabile inserimento nella maggioranza parlamentare e successivamente nel governo (Mattina 1997). Tali fattori, che contribuiscono ad una più netta divisione dei ruoli, non sussistono nel caso delle associazioni vicine alla Dc e dei sindacati autonomi. Al contrario, l'adozione di strategie politiche improntate ad una forte autonomia dei gruppi viene in questo caso ulteriormente scoraggiata dalla richiesta di sostegno, in termini elettorali e di dirigenti professionalizzati da inserire nei governi locali, che Forza Italia avanza ai gruppi stessi. Alle fortune elettorali nazionali del movimento non corrisponde, infatti, un'adeguata ramificazione organizzativa, né un appropriato sistema di raccordo con la periferia, condizioni fondamentali per selezionare una leva di candidati capaci di competere, al livello locale, con le stesse probabilità di successo con cui il partito compete al livello nazionale. Lo scarso radicamento organizzativo del movimento ha così spinto le articolazioni periferiche di FI a cooptare estesamente i gruppi dirigenti delle associazioni di categoria di ispirazione moderata e dei sindacati autonomi, sperando con ciò di far breccia nell'elettorato corrispondente e di reclutare una leva di amministratori già collaudati e perciò capaci di imprimere una spinta anche alla nascente struttura periferica. Il risultato di queste diverse sollecitazioni è una proliferazione anomala di rappresentanti dei gruppi di interesse economico fra le file degli eletti di FI, e l'instaurazione di un rapporto di reciproca strumentalizzazione fra gruppi e partito, fondato sulla debolezza organizzativa di entrambi i soggetti.

\section{Dalla rappresentatività alla rappresentanza}

Fino a che punto è possibile stabilire un parallelismo fra estrazione sociale e rappresentanza politica partendo dalla composizione professionale degli eletti? Stabilito che i veri benefi- 
TAB. 8. Graduatoria degli attori sociali ed economici in base all'attenzione loro accordata dagli eletti di Forza Italia

\begin{tabular}{lcc}
\hline Gruppi & Punteggio & Distacco fra i due gruppi successivi \\
\hline Piccoli imprenditori & 2,42 & - \\
Giovani & 2,37 & $-0,05$ \\
Artigiani & 2,36 & $-0,01$ \\
Liberi professionisti & 2,31 & $-0,05$ \\
Commercianti & 2,30 & $-0,01$ \\
Disoccupati & 2,09 & $-0,21$ \\
Donne & 1,96 & $-0,13$ \\
Operai & 1,92 & $-0,04$ \\
Pensionati & 1,79 & $-0,13$ \\
Casalinghe & 1,72 & $-0,07$ \\
Impiegati & 1,69 & $-0,03$ \\
Intellettuali & 1,55 & $-0,14$ \\
Grandi imprenditori & 1,41 & $-0,14$ \\
\hline
\end{tabular}

Nota: Il testo della domanda era il seguente: «Ogni rappresentante sente di avere legami particolari con alcune categorie di cittadini, e meno stretti con altri. Quanto strettamente sente di rappresentare gli interessi delle seguenti categorie di cittadini?». La scala proposta nel questionario prevedeva tre possibili risposte in ordine di intensità decrescente, ciascuna distanziata di una unità. L'indice adottato può così variare fra un massimo di 3 ed un minimo di 1 . I punteggi riportati sono i valori medi corrispondenti alle risposte fornite.

ciari dell'offerta di rappresentanza non possono essere individuati che sul terreno delle policies, il questionario somministrato ci consente di conseguire un risultato intermedio mediante il confronto fra i dati relativi alla rappresentanza sociologica e alla presenza dei gruppi di interesse e la mission rappresentativa che gli eletti si attribuiscono, vale a dire la vocazione a rappresentare raggruppamenti specifici e riconoscibili di interessi. A tale proposito, con l'ausilio di una scala graduata, è stato richiesto agli intervistati di indicare, per una ampia serie di categorie sociali e professionali, l'intensità con la quale ritengono di rappresentarle. Le risposte fornite sono state poi tradotte in punteggi sintetici tali da consentire la comparazione e la definizione di un ranking decrescente che si espone in tabella 8.

I dati contenuti in tabella 8 attestano un notevole grado di continuità con quelli commentati alle pagine precedenti. Osserviamo, infatti, che la peculiare composizione sociale del campione, e la costellazione dei gruppi di interesse in esso rappresentati, si riflettono molto nettamente anche a livello di auto-rappresentazione di ruolo, dal momento che i settori professionali maggiormente presenti sono quelli che gli eletti ritengono di 
rappresentare con più forza. Il dato che emerge con maggiore chiarezza è l'assoluto primato delle categorie imprenditoriali e professionali su tutte le altre, in piena concordanza con quanto emerso dall'esame delle appartenenze associative e delle professioni svolte. Con la parziale eccezione dei giovani e dei disoccupati, che ogni politico dotato di buon senso non può mancare di menzionare in un paese che lamenta un tasso di disoccupazione pari al $12 \%$ della popolazione attiva, appare del tutto marginale il favore accordato ai restanti gruppi sociali ed in particolare alle varie categorie di non attivi quali casalinghe e pensionati. Se è pur vero che è da questi settori che proviene una parte comunque non piccola dei voti raccolti da Forza Italia (Mannheimer 1994; Gray e Howard 1995), non altrettanto si può dire circa la provenienza dei suoi amministratori locali, praticamente assenti in queste fasce sociali (tab. 7). Rileviamo perciò solo dei limitati scostamenti fra rappresentanza sociologica e rappresentanza politica dichiarata: coloro che risultano trascurati dalla prima lo sono anche dalla seconda e viceversa. Il dato non fa altro che confermare, per lo meno sul terreno dei comportamenti attesi e delle intenzioni esplicite, le ipotesi iniziali circa la contiguità esistente fra origine sociale e rappresentanza politica.

Vi sono, tuttavia, alcune articolazioni di quest'ipotesi generale che vale la pena commentare in quanto particolarmente indicative del rapporto fra movimento politico e referenti sociali $\mathrm{e}$, in particolare, della frammentazione interna al blocco delle classi medio-alte da cui origina la peculiarità dell'offerta politica di Forza Italia. Il dato più importante, ma forse non inatteso, consiste nell'esistenza di una netta discriminante a favore della piccola imprenditoria a fianco di un vero e proprio ostracismo nei confronti della grande. A ciò si associa una forte attenzione anche per gli altri settori economici caratterizzati dalla piccola dimensione aziendale (artigiani, commercianti) che abbiamo visto essere ben presenti, almeno in termini relativi, nella tabella sulla professione degli eletti. Lo stesso mondo delle libere professioni, egemone per quanto riguarda la provenienza professionale, vede ridotto il suo peso a vantaggio di queste categorie quando si passa all'offerta esplicita di rappresentanza. Ciò significa che i liberi professionisti del campione, o buona parte di essi, indicano come principali referenti altre categorie socioeconomiche, tendendo opportunamente a superare la propria specificità professionale, poco spendibile in termini di elettorato di riferimento. Un comportamento peraltro che non si disco- 
sta troppo da quello del funzionariato pubblico-partitico della Prima repubblica. La sovrarappresentazione delle libere professioni, per quanto riguarda la rappresentanza sociologica, sembra così esplicarsi anche e soprattutto in funzione di servizio delle istanze dei ceti medi ed imprenditoriali nei cui confronti, peraltro, viene svolta la normale attività professionale, stabilendo così una continuità dei rapporti di scambio che già sussistono nell'arena economica.

Un esame dettagliato di tabella 8 ci consente di meglio precisare queste ipotesi. I distacchi fra le varie categorie del ranking, riportati nella seconda colonna, sono per lo più ridotti. Si registrano tuttavia alcune fratture dell'andamento incrementale che sono significative perché a ciascuna di esse corrisponde l'identificazione di sottogruppi tendenzialmente omogenei quanto a intensità della rappresentanza offerta. Segnaliamo, in particolare, la prima frattura, che separa il grande gruppo dei professionisti e dei ceti medi imprenditoriali dai disoccupati $(-0,21)$, e l'ultima frattura, che separa i già negletti intellettuali dai grandi imprenditori $(-0,14)$, che sono l'unico gruppo con un punteggio inferiore ad una teorica «sufficienza» (1,5 punti). Se limitiamo l'analisi alle sole categorie degli attivi, osserviamo che si apre un vero e proprio baratro ( $-0,34$ punti) fra i commercianti e gli operai, mentre il divario fra piccoli e grandi imprenditori è addirittura superiore ad 1 . Il gruppo liberi professionisti + ceti medi imprenditoriali (prime cinque posizioni del ranking), a differenza delle altre categorie, si caratterizza per l'elevato punteggio e per la relativa omogeneità dello stesso fra le categorie componenti; lo si può dunque effettivamente isolare come gruppo privilegiato ed egemone nella destinazione dell'offerta di rappresentanza degli eletti.

La vocazione rappresentativa di Forza Italia si indirizza quindi in prevalenza verso il cosiddetto «settore tradizionale», confermando così alcune delle ipotesi avanzate da Berger e Piore (1982) circa l'importanza delle variabili politiche nella definizione e promozione degli interessi di questo comparto e circa la sua centralità nella composizione dell'elettorato dei partiti conservatori e moderati europei. Osserviamo, dunque, che Forza Italia si è fatta garante della copertura politica delle istanze dei ceti medi imprenditoriali che nel nostro paese si sono tradizionalmente qualificati per una minore autonomia di mercato e per una diretta dipendenza nei confronti di provvidenze pubbliche, di volta in volta incarnatesi in comportamenti positivi 
(leggi di incentivazione) od omissivi (in tema di fiscalità, sicurezza del lavoro e dell'ambiente) delle autorità e la cui reiterazione nel tempo era garantita dall'inamovibilità della Dc dalla guida del paese. La contropartita offerta consiste - oggi come un tempo - nella mobilitazione in favore del movimento di settori sociali ed economici che, per quanto minacciati, sono ben lungi dal perdere consistenza demografica e che, per certi aspetti, continuano a manifestare importanti segnali di vitalità (Davico 1994; Belloni 1994). Di più, le associazioni di rappresentanza di tali gruppi sociali costituiscono un vivaio di professionismo politico, per lo meno potenziale, presso il quale Forza Italia ha attinto una parte significativa dei suoi quadri dirigenti locali, promuovendo così la crescita della loro diretta presenza nelle assemblee elettive, in contrasto con la natura umbratile dell'intervento dei gruppi nel processo politico.

\section{Conclusioni}

Alla luce dei dati commentati possiamo affermare che Forza Italia costituisce un fenomeno realmente innovativo nel sistema politico italiano anche a livello locale, sia pure nei limiti che abbiamo evidenziato. L'accesso di Forza Italia nelle istituzioni del governo locale ha implicato un radicale rovesciamento dei riferimenti tradizionalmente impiegati per descrivere le élites politiche periferiche. Sia sotto l'aspetto del reclutamento che della rappresentanza sociale, gli eletti di Forza Italia mostrano evidenti e cospicue differenze con le precedenti leve di amministratori locali. Quanta parte di queste differenze sia imputabile a consapevoli ed autonome scelte della dirigenza del partito, ovvero alla fase di transizione del sistema partitico, non è dato sapere dai risultati del questionario. Tuttavia, l'evidenza empirica raccolta ci consente di proporre un primo e provvisorio nucleo di conclusioni.

Assumendo come termine di paragone la coalizione di forze politiche e di interessi sociali dominante nel sistema politico italiano fino ai primi anni Novanta, ed in particolare la Dc che di tale coalizione fu il perno, gli eletti di Forza Italia manifestano una netta continuità per orientamento ideologico e settori sociali prediletti. Ciò che muta è invece il modello di reclutamento dei politici locali, a cui ha finito per corrispondere una sensibile alterazione della composizione sociologica degli eletti e una cre- 
scita del ruolo dei gruppi di interesse. In altre parole, la crisi ed il mutamento del sistema partitico italiano hanno creato un vuoto nel precedente sistema di selezione delle classi dirigenti del centro-destra. Un vuoto che, per adesso, Forza Italia ha colmato in due modi: da un lato, impiegando la parte meno compromessa e anagraficamente più giovane delle vecchie élites; dall'altro, proponendosi come benevolo e interessato recettore dell'attivismo di gruppi sociali ed economici rimasti privi di un referente politico credibile. Ciò ha dato luogo a processi di selezione a forte impianto societario, al cui interno il partito ha mantenuto, per scelta e per forza di cose, un ruolo meno attivo che in passato e i cui principali esiti sono stati l'ampio accesso dei gruppi di interesse e delle professioni a forte visibilità sociale. I due meccanismi non sono del tutto alternativi, ma tendono a giustapporsi dando vita ad un modello sincretico che contiene al proprio interno elementi potenzialmente contraddittori. Tutto ciò ha fatto emergere un gruppo di amministratori locali eterogeneo e, nonostante ciò, ben determinato quanto a origine sociale e composizione socio-anagrafica. Per ognuno di essi l'adesione al partito costituisce, come è ovvio, un requisito essenziale e per molti di essi si rileva una qualche forma di precedente attività politica, sia pure marginale. Tutti gli eletti del campione però, vuoi per la professione svolta, vuoi per i reticoli associativi di cui sono partecipi, si collocano in una posizione centrale rispetto alla struttura sociale delle comunità locali di riferimento. Da questo punto di vista, dunque, Forza Italia si qualifica per essere l'attore politico più adatto a raccogliere le spinte partecipative che questa centralità sociale determina (Milbrath 1965).

Per quanto riguarda l'offerta di rappresentanza, ricordiamo che l'immagine concordemente fissata da tutte le ricerche sulla classe politica locale precedenti al 1994 è quella di un'élite professionale sociologicamente distaccata dalla società che rappresenta, a prescindere dalla collocazione ideologica delle varie formazioni politiche. Ciò si inverava nella marginalizzazione strutturale sia dei segmenti socio-anagrafici più deboli, anche se quantitativamente rilevanti (donne e giovani), sia delle categorie professionali più direttamente coinvolte nella produzione di ricchezza (operai, imprenditori, artigiani). Finiva perciò per prevalere una sorta di «nuova classe» composta dal funzionariato, in prevalenza pubblico o partitico.

L'esame congiunto dei dati relativi alle variabili socio-anagrafiche, professionali ed associative degli eletti di FI conferma 
solo in parte questo modello. Forza Italia, infatti, da un lato replica la marginalizzazione delle categorie sociali deboli, che anzi vengono ancor più penalizzate che nel passato, mentre dall'altra ribalta completamente il quadro relativo alla professione degli eletti. Anziché ingrossarsi di funzionari, le liste forziste attingono a piene mani dal mondo delle professioni, dal commercio e dalle attività produttive tradizionali, in ossequio sia ai precetti programmatici che in risposta all'autonoma mobilitazione di questi ceti. Il risultato è che il movimento finisce per rappresentare, dal versante economico e demografico, solo la parte emergente, e nemmeno tutta, dell'iceberg sociale.

Una configurazione socio-anagrafica come quella rilevata risulta del resto congruente con le teorie classiche della partecipazione politica che la pongono in diretta correlazione con lo status sociale (Milbrath 1965). Ciò che stupisce è semmai la portata del fenomeno e la sua nettezza. L'ipotesi che per il momento sembra più plausibile è la seguente. Esiste in tutte le democrazie di mercato una costante tendenza all'ipertrofia dei segmenti sociali centrali nei posti di potere. In Italia, tuttavia, questa tendenza è stata potentemente contrastata dall'esistenza di partiti di massa organizzati i quali, riuscendo ad affermarsi come unici regolatori dell'accesso alle sedi politiche (gatekeepers), hanno imposto per decenni propri requisiti d'accesso (carriera partitica, fedeltà, scuole di partito), attenuando la pressione delle spinte societarie. Il risultato è quella tendenziale omogeneizzazione dei percorsi di accesso e delle origini sociali delle élites partitiche italiane da molti descritta e criticata. La lunga crisi del partito di massa, aggravata in Italia dal problematico mutamento del sistema partitico, rende meno cogenti questi vincoli nell'area di centro-destra in cui la crisi dei vecchi partiti è particolarmente grave. Il nuovo soggetto politico che deliberatamente intende occupare quest'area è però privo della capacità di imporre un proprio modello di reclutamento. Esso, pertanto, accoglie e sollecita senza eccessivi condizionamenti lo spontaneo attivismo dei segmenti sociali rimasti privi di riferimento e minacciati dai processi di modernizzazione del mercato. Il risultato è un modello di reclutamento a prevalente controllo societario, il quale dà luogo ad una offerta di rappresentanza che della società stessa finisce inevitabilmente per replicare le disuguaglianze. L'unica importante eccezione è qui costituita dall'esclusione della grande impresa dal gruppo degli attori sociali meritevoli di interesse da parte di FI, un'esclusione, o meglio 
una marginalizzazione, che si spiega sia con la maggiore autonomia di Confindustria rispetto alle organizzazioni concorrenti, ed il conseguente minor bisogno di tutela, sia con la tradizionale scarsa inclinazione dei grandi imprenditori ad entrare direttamente nell'arena politica (Mattina 1991).

Per quanto riguarda il dettaglio dei gruppi sociali maggiormente rappresentati, l'analisi comparativa dei tre indicatori adottati (professione, affiliazione, concezione del proprio ruolo) fornisce un quadro di sostanziale coerenza fra origine sociale, rappresentanza associativa e mission rappresentativa. Nello specifico, tuttavia, possiamo individuare delle variazioni che attestano l'influenza esercitata dal particolare modello di reclutamento. In questo contesto, la sovraesposizione politica delle libere professioni si associa ad una sorta di delega da parte dei ceti medi imprenditoriali meno inclini all'impegno diretto in politica. In ciò si ricalca uno schema di rapporto fra partito e ceti medi già esistente al tempo della $\mathrm{Dc}$, con la rilevante eccezione costituita dalla sostituzione dei liberi professionisti ai funzionari pubblici e di partito quale figura cardine della regolazione degli scambi fra $\mathrm{i}$ due soggetti. Quanto detto, assieme alla apparente ostilità nei confronti della grande impresa, attesta la perdurante centralità del settore economico tradizionale nella strutturazione dell'offerta politica del centro-destra in Italia, in particolare per quanto concerne la fissazione dei rapporti di delega politica, la configurazione istituzionale degli assetti economici e, last but not least, la stabilizzazione di un consenso di massa in favore dei partiti moderati o conservatori. In base a ciò FI potrebbe ragionevolmente mirare a proporsi, come esplicitamente indicato nel congresso di Milano, quale nuova Dc, erede non solo della sua collocazione politica, ma anche dei gruppi sociali di riferimento. Tuttavia, il dna politico degli eletti di Forza Italia, sia in termini di composizione sociale che di preferenze manifestate quanto a rappresentanza dei settori sociali, risulta scarsamente dotato di quelle prerogative di interclassismo che hanno fatto la fortuna della Dc, consentendole di occupare per decenni una posizione centrale all'interno del sistema partitico e nella politica degli interessi organizzati. La collocazione ideologica e l'esplicita offerta di rappresentanza di Forza Italia, al contrario, se pure la consolidano nel ruolo di portabandiera di settori economici e sociali che risultano decisivi ai fini di un effettivo radicamento nel tessuto sociale del paese, al tempo stesso sembrano ridimensionarne ab initio le aspi- 
razioni ad affermarsi quale futuro baricentro del sistema politico italiano. A questo proposito non si può fare a meno di notare che la rinuncia, almeno apparente, a tarare la propria offerta di rappresentanza anche sulle esigenze della grande impresa e del lavoro dipendente rischia di costringere il partito in una sorta di «ghetto rappresentativo», al cui interno potrà prosperare ma non raggiungere la massa critica necessaria ad occupare il baricentro del sistema partitico. In conseguenza di ciò, le più serie opzioni di crescita elettorale stanno oggi, per FI, al di fuori del suo bacino rappresentativo «naturale» e consistono, essenzialmente, nel residuo carisma del proprio leader e nell'impiego massiccio di strumenti di propaganda capaci di attrarre un consenso tendenzialmente interclassista.

\section{Riferimenti bibliografici}

Almond, G. e S. Verba (1963), The Civic Culture: Political Attitudes and Democracy in Five Nations, Princeton, Princeton University Press.

Barberis, C. (1988), La classe politica municipale, Milano, Angeli.

Bartolini, S. e R. D'Alimonte (a cura di) (1995), Maggioritario ma non troppo. Le elezioni politiche del 1994, Bologna, Il Mulino.

Belloni, M.C. (1994), Un paese di imprenditori piccoli piccoli, in Ginsbourg (1994), pp. 236-238.

Bequart Leclercq, J. (1976), Paradoxes du pouvoir local, Paris, Presse de la Fondation Nationale des Sciences Politiques.

Berger, S. e M. Piore (1982), Dualismo economico e politica nelle società industriali, Bologna, Il Mulino.

Bettin, G. (a cura di) (1988), Classe politica e città, Padova, Cedam.

Bettin, G. e A. Magnier (1989), Il consigliere comunale, Padova, Cedam.

Cotta, M. (1986), Parlamenti e rappresentanza, in Pasquino (a cura di), pp. 281-328.

Davico, L. (1994), Il mondo eterogeneo dei ceti medi, in Ginsbourg (1994), pp. 234-235.

D'Alimonte, R. e S. Bartolini (a cura di) (1997), Maggioritario per caso. Le elezioni politiche del 1996, Bologna, Il Mulino.

Diamanti, I. (1995), I dirigenti delle associazioni culturali nel Mezzogiorno: caratteri sociali, modelli di partecipazione e orientamenti di valore, in «Meridiana», n. 22-23, pp. 185-221.

Diamanti, I. e R. Mannheimer (a cura di) (1994), Milano a Roma. Guida all'Italia elettorale del 1994, Roma, Donzelli.

Ferrante, M. (1998), Transizione di regime e interessi imprenditoriali in Italia, in «Rivista Italiana di Scienza Politica», n. 1, pp. 81-118. 
Ginsbourg, P. (a cura di) (1994), Stato dell'Italia, Milano, Il Saggiatore-Bruno Mondadori.

Gray, L. e W. Howard (1995), Forza Italia. Il partito americano, in M. Fedele e R. Leonardi (a cura di), La politica senza i partiti, Roma, Seam, pp. 95-106.

Key, V.O. (1961), Public Opinion and American Democracy, New York.

Maraffi, M. (1995), Forza Italia, in G. Pasquino (a cura di), La politica italiana. Dizionario critico 1945-1995, Roma-Bari, Laterza, pp. 247-259.

- (1996), Forza Italia dal governo all'opposizione, in M. Caciagli e D.I. Kertzer (1994), Politica in Italia. I fatti dell'anno e le interpretazioni. Edizione '96, Bologna, Il Mulino, pp. 139-157.

Mannheimer R. (1994), Forza Italia, in Diamanti e Mannheimer (1994), pp. 29-42.

Mastropaolo, A. (1993), Il ceto politico. Teoria e pratica, Roma, Nis.

Mattina, L. (1991), Gli industriali e la democrazia, Bologna, Il Mulino.

- (1994), Gli attori economici. Vincitori e vinti, in Diamanti e Mannheimer (1994), pp. 151-158.

- (1995), I candidati, in Bartolini e D'Alimonte (1995), pp. 233-270.

- (1997), Partiti e sindacati nella crisi della democrazia italiana: dal dominio alla simbiosi, in «Studi politici», 1, pp. 1-56.

Mattina, L. e A. Tonarelli (1997), I candidati. Visioni politiche e carrie$r e$, in D'Alimonte e Bartolini (1997), pp. 35-69.

Milbrath, L.W. (1965), Political Participation, Chicago, Rand McNally.

Morlino, L. (a cura di) (1991), Costruire la democrazia, Bologna, Il Mulino.

Morlino, L. (1996), Crisis of Parties and Change of Party System in Italy, in «Party Politics», n. 1, pp. 5-30.

Pasquino, G. (1986), Partecipazione politica gruppi e movimenti, in $\mathrm{Pa}$ squino (a cura di), pp. 191-230.

Pasquino, G. (a cura di) (1986), Manuale di scienza della politica, Bologna, Il Mulino.

Ramella, F. (1994), Gruppi sociali e cittadinanza democratica, in «Meridiana», n. 20, pp. 93-133.

Recchi, E. (1998), Giovani politici, Padova, Cedam.

Sills, D.L. (1972), Voluntary Associations. Sociological Aspects, in D.L. Sills (a cura di), International Encyclopedia of the Social Sciences, New York, Macmillan-The Free Press.

Verba, S. e N.H. Nie (1972), Participation in America. Political Democracy and Social Equality, Chicago, The University of Chicago Press.

Verzichelli, L. (1997), La classe politica della transizione, in D'Alimonte e Bartolini (1997), pp. 309-350. 